\title{
The fallacy of placing confidence in confidence intervals - A commentary
}

Jose D. Perezgonzalez

\begin{abstract}
'The fallacy of placing confidence in confidence intervals' (Morey et al., 2016, Psychonomic Bulletin \& Review, doi: 10.3758/s13423-015-0947-8) delved into a much needed technical and philosophical dissertation regarding the differences between typical (mis)interpretations of frequentist confidence intervals and the typical correct interpretation of Bayesian credible intervals. My contribution here partly strengthens the authors' argument, partly closes some gaps they left open, and concludes with a note of attention to the possibility that there may be distinctions without real practical differences in the ultimate use of estimation by intervals, namely when assuming a common ground of uninformative priors and intervals as ranges of values instead of as posterior distributions per se.
\end{abstract}

\footnotetext{
"The Authority: 'You must not use confidence intervals; they are discredited!' Practicing Statistician: 'I use confidence intervals because they correspond exactly to certain needs of applied work.'” (Neyman, 1977, p. 98)
}

Morey, Hoekstra, Rouder, Lee, and Wagenmakers $\left(2016^{1}\right)$ delve into a much needed technical and philosophical dissertation regarding the differences between typical (mis)interpretations of frequentist confidence intervals and the typical correct interpretation of Bayesian credible intervals. My contribution here partly strengthens Morey et al.'s argument, partly closes some gaps they left open, and concludes with a note of attention to the possibility that there may be distinctions without real practical differences in the ultimate use of estimation by intervals, namely when assuming a common ground of uninformative priors and intervals as ranges of values instead of as posterior distributions per se.

I will break down my commentary into relevant sections, starting with definitions, followed by coverage intervals, countable intervals, credible intervals and final notes. As far as practicable, I will quote Morey et al. before commenting, so that the article gets a clearer cue-response structure.

\footnotetext{
${ }^{1}$ Morey, R. D., Hoekstra, R., Rouder, J. N., Lee, M. D., \& Wagenmakers, E. J. (2016). The fallacy of placing confidence in confidence intervals. Psychonomic Bulletin \& Review, 23, 103-123. doi: 10.3758/s13423-0150947-8
} 


\section{Definitions}

"It is therefore helpful to differentiate a [confidence] procedure ... from a confidence interval: an X\% confidence procedure is any procedure that generates intervals [which will] cover (sic) [the parameter] in X\% of repeated samples, and a confidence interval is a specific interval generated by such a process" (Morey et al., 2016, p. 104).

Morey et al. make a good effort in differentiating between the process of generating a confidence interval $(\mathrm{Cl})$ and the output of such process. Illustrating with a simpler example, the tossing of a fair six-faced die is a procedure for generating a single outcome out of six faces the die can come up. The example of a die is unrelated to Cls but we can reasonably use it to better understand Morey et al.'s argument: confidence procedures and confidence intervals are two different things.

Furthermore, a stochastic, random element can be ascribed to the former, such that initial probabilities may be possible. For example, an output such as 'Any face other than a six' has probability $5 / 6$ (or $83 \%$ ) of occurring. Equally, the output 'No other face but a six' will occur with probability $1 / 6$ (or $17 \%$ ). Similarly, we can run our confidence procedure so as for generating intervals with a given probability $(80 \%, 95 \%, 99 \%)$ of capturing the parameter of interest.

People familiar with dice, however, know the probability of the outcome without having to resort to repeated sampling. Assuming the die is fair and the tossing allows for randomness, each face ought to come up with equal probability, so that we may take those probabilities into our calculations: We do not need repeated tossings for ascertaining the probability of a fair die. Although we are not limited in our opportunity for testing for fairness-e.g. via balance testing (Vetanen, 2016 ${ }^{2}$ ) or repeated tossings (Haran, 2016 ${ }^{3}$ )-we can be satisfied that probabilities are inherent to the construction of symmetric dice; that is, inherent to a physical model dependent on the construction of the object in question (thus, probability as "equal possibilities based on physical symmetry", Gigerenzer et al, 1989, p. $7^{4}$; or as known unknown risks, Ellenberg, $2014^{5}$ ). Similarly, most Cls are obtained via statistical models, reduced to resolving mathematical formulas or to following established procedures rather than resorting to repeated sampling. Here I do not deny that statistical models may have been born out of observed regularities in the distribution of data under repeated sampling, nor do I deny that such properties can be found using empirical methods (bootstrapping being an example). What I am saying is that repeated sampling per se does not need to occur for generating a $\mathrm{Cl}$ when assuming a statistical model.

In fact, it may also be necessary to clarify what repeated sampling actually means, then label Cls accordingly. In the argumentation presented on page 104, repeated sampling seems to accord to the concept of resampling, which is what bootstrapping does empirically but also what underlies the mathematical generation of theoretical sampling distributions from statistical models (I shall refer to these as coverage procedures and coverage intervals). On the other hand, on page 106 it is

\footnotetext{
${ }^{2}$ Vetanen, M. (2016). Spirit Mountain Casino Dice balance testing. Retrieved from https://youtu.be/JDJJtt4R1JU

${ }^{3}$ Haran, B. (Producer). (2016). Fair Dice (Part 2) - Numberphile. Retrieved from https://youtu.be/8UUPIImm0dM

${ }^{4}$ Gigerenzer, G., Swijtink, Z., Porter, T., Daston, L., Beatty, J., \& Krüger, L. (1989). The empire of chance. How probability changed science and everyday life. Cambridge, UK: Cambridge University Press.

${ }^{5}$ Ellenberg, J. (2014). How not to be wrong. The hidden maths of everyday life. London, UK: Penguin Books.
} 
associated with the long-run, which brings with it a temporal element to sampling and which is typically depicted as a column of independent Cls obtained in sequence (e.g., Neyman, 1977; Cumming, 2012; also Cumming's dance of $\left.\mathrm{Cls}, 2016^{6}\right)$ (I shall refer to these as countable procedures and countable intervals).

Using the example of a fair die again, while its probabilities are inherent to the model (the physical die), we expect such probabilities to also appear under resampling (e.g., if testing the fairness of the die in an experiment) as well as in the long-run (i.e., if testing the fairness of the die in several experiments). In fact, we could even think of this as a 'nested' property, with model probabilities nested within resampling nested within long-run behaviour. Such nesting implies two things: On the one hand, the nesting goes in one direction only, from model to resampling to long-run propertiesthus, the probabilities expected at one level are also expected at a higher level-yet not all lower levels may be possible-for example, bootstrapping allows for resampling when the assumptions of a statistical model go unfulfilled. On the other hand, a parsimonious definition of probability cannot exclude the smallest nesting possible. Nesting allows for long-run probabilities to be the only necessary condition yet the probabilities derived from a statistical model are in themselves sufficient. Indeed, Neyman refers to both resampling and the long-run as justifications for recommending confidence procedures, not as definitional properties per se (Neyman, 1937).

The definition of Morey et al. stops at resampling, thus, in principle, excluding probabilities derived from statistical models and resulting in a less than parsimonious definition. We could, of course, reasonably argue that resampling assumes theoretical resampling, but the point is made that actual resampling, whether theoretical or empirical, may not need to occur, the statistical model being "an idealized generating mechanism that could have given rise to data", yet "the assigning of probabilities to legitimate (generic) events in model-based induction does not invoke the [resampling or] 'long-run' metaphor. Indeed, there are no conceptual or technical difficulties in assigning a probability to single events ... in the context of [a statistical] model" (Spanos, $2013^{7}, \mathrm{p}$. 1557, 1570).

"A confidence procedure is a random process" (Morey et al., 2016, p. 104).

A die is not random in itself: It simply is a tool that favours randomness when also considering related issues, such as the construction of the die (fair or biased), the quality of the tossing, the surface where the die is tossed onto, etc. The tossing procedure, thus, incorporates all that allows an outcome to occur. Similarly, if randomness is required for calculating a $\mathrm{Cl}$, it rests on issues such as the selection of the sample, the cleaning of the data, the particular procedure used for generating $\mathrm{Cls}$, etc. Thus randomness itself is not necessary for, and it may not be guaranteed by, the procedure of simply generating a $\mathrm{Cl}$ (e.g., randomness is not included in Neyman's quote given as Definition 1; meanwhile, randomness refers to the dataset, not to the calculus of Cls in Neyman 1937, 1977).

\footnotetext{
${ }^{6}$ Cumming, G. (2016). 7.4 p values, for ITNS book. Retrieved from https://youtu.be/3FjoUOolOYQ

${ }^{7}$ Spanos, A. (2013). A frequentist interpretation of probability for model inductive inference. Synthese, 190, 1555-1585. doi:10.1007/s11229-011-9892-x
} 
"A confidence interval is a specific interval ... [it] is observed and fixed. . . Frequentist $\mathrm{Cl}$ theory says nothing at all about the probability that a particular, observed confidence interval contains the true value; it is either 0 (if the interval does not contain the parameter) or 1 (if the interval does contain the true value)" (Morey et al., 2016, p.104, 105).

Indeed, once the die is rolled and has come to a rest, any initial probabilities in the procedure will collapse into the certainty of the observed outcome. One thing we need to be wary, however, is that the $\mathrm{Cl}$ is not the outcome that we seek, but a mere output towards that outcome. What we seek is to estimate the population parameter, not merely calculate, for example, a descriptive interval of the sampling distribution. As Neyman put it,

The basic formula [for the frequently correct confidence coefficient] is written not in terms of the observed [sample data] but in terms of the observable [population data].... If one substitutes in [the formula] the observed [sample data] in the place of the observable [population data], the result would be absurd ... The probability ... [ought to be] zero ... [or] unity, neither of any interest. (1977, p. 118, 119)

In research we do not have the privilege of observing the parameter, and the $\mathrm{Cl}$, being an inferential statistic, cannot be its substitute. Thus, we are in a situation where a curtain blocks our view of the die. Before unveiling the curtain, what probability do we give to the result being 'Any face other than a six'? We know the probabilities of the procedure, but the die has already been tossed, so they may not apply. We also know that the die has come to a rest and there is no changing the outcome, so the probability of the outcome has to be either ' 1 ' or ' 0 '. In this context, it seems reasonable to ascribe to the unveiled die the initial probabilities of the procedure and hedge our bets accordingly. A similar thought process seems reasonable with Cls understood as intermediate outputs, the probability of the confidence coefficient being an expectation. As Neyman put it, "the probability statements refer to the problem of estimation with which the statistician will be concerned in the future" (Neyman, 1937, p. 349); thus, not to a measure of final precision (Mayo, 1981; also Savage, $\left.1962^{8}\right)$.

"Of the many kinds of interval estimates, the most popular is the confidence interval. ... Alternatives - such as Bayesian credible intervals ...-are not as commonly used" (Morey et al., 2016, p.103, 107).

A final comment where our dice example is helpful is in illustrating the role of Bayesian credible intervals and their relationship to Cls. We have so far assumed the die is fair and its tossing allows for a random outcome. This assumption is, however, a belief that we are not obliged to accept. We may prefer to 'keep an open mind' regarding its fairness, or we may suspect bias in the procedure. $\mathrm{Cls}$ - a product of Frequentism - provide no way of accounting for beliefs other than, for example, the (uniform) assumption that a die is fair. Earlier I talked about frequentist-based probabilities being nested into higher-level environments (statistical model - resampling - long-run behaviour), a feature which does not prevent Frequentism itself from being nested into a higher-level environment that allows for beliefs other than models' assumptions to be taken into account.

\footnotetext{
${ }^{8}$ Savage, L.J. (1962). The foundations of statistical inference. London, UK: Methuen.
} 
Bayesian statistics is such higher-level environment, capable of subsuming frequency statistics within the likelihood apparatus of its logic. Thus, not only one should expect coverage intervals to numerically correspond to credible intervals under certain Bayesian conditions (such as when using uniform priors or under the principle of stable estimation, Phillips, $1973^{9}$; also Edwards, Lindman, \& Savage, $1963^{10}$ ), but this should be taken as a consequence of such nesting and not merely as a "hand-waving defense of confidence intervals" (Morey et al., 2016, p. 119). Thus, a Bayesian can, under certain inferential conditions, consider a coverage interval to be a credible interval (a.k.a., it shows sufficient, although not necessary, likelihood for such interpretation). The opposite is not true, and a frequentist confidence interval cannot inherit higher-level Bayesian interpretations it does not possess.

"These all involve reasoning about the parameter from the observed data: that is, they are 'post-data' inferences... The key confusion ... is the confusion of what is known before observing the data-that the $\mathrm{Cl}$, whatever it will be, has a fixed chance of containing the true value - with what is known after observing the data" (Morey et al., 2016, p. 104, 105).

When dealing with data testing, Frequentism tests the probability of the data given a hypothesis while Bayesianism tests the probability of a hypothesis given the data. This differentiation carries into estimation by intervals when we consider that a coverage interval (as the bulk of the distribution) substitutes a test (on the tails), and that the hypothesis of reference is not a hypothesis external to the sample $\left(H_{0}\right)$ but inherent to the sample itself: the research hypothesis $\left(H_{R}: M=\mu_{x}=\right.$ $\mu)$. Thus, a coverage interval informs about the probability of an interval assuming the statistic equals the parameter $\left[p\left(C I \mid M=\mu_{x}=\mu\right)\right.$, or $\left.p\left(D \mid H_{R}\right)\right]$, while a credible interval informs about the probability of the research hypothesis given the interval $\left[p\left(M=\mu_{x}=\mu \mid C I\right)\right.$, or $\left.p\left(H_{R} \mid D\right)\right]$.

This understanding helps resolve Morey et al.'s "violence" towards the temporal approach to 'before' and 'after' observing the data (also Mayo, $1981^{11}$ ), which seems to me artificial and devoid of empirical realization. Frequentists as well as Bayesians are allowed to observe the sample's data, have knowledge before observing the data as well as make inferences after observing the data, for estimation by intervals is but estimation based on certain assumptions, not on visual observation. What differentiates Frequentism and Bayesianism is that the former only looks onto the probability of the data given the statistic, while the later allows for also contemplating other information as prior probability. Either should be stable enough without having to resort to an artificial temporal separation between 'before' and 'after' observing data.

\footnotetext{
${ }^{9}$ Phillips, L.D. (1973). Bayesian statistics for social scientists. London, UK: Nelson and Sons.

${ }^{10}$ Edwards, W., Lindman, H., \& Savage, L. J. (1963). Bayesian statistical inference for psychological research. Psychological Review, 70, 193-242.

${ }_{11}$ Mayo, D. (1981). In defense of the Neyman-Pearson theory of confidence intervals. Philosophy of Science, 48(2), 269-280.
} 


\section{Coverage intervals}

"The name 'confidence interval'-rather than, for instance, the more accurate 'coverage procedure'-encourages the Fundamental Confidence Fallacy" (Morey et al., 2016, p. 105).

We have so far established that the difference between pre-data and post-data does not accord to empirical evidence. We also established that we never have the benefit of hindsight so that $\mathrm{a} \mathrm{Cl}$ is a probabilistic intermediate output (a parameter estimation) not the final outcome (a parameter known with certainty). Finally, it is reasonable to ascribe to the output the probabilities of the procedure. Therefore, it would be accurate to use the label 'coverage interval' to refer to the observed output (the actual interval), and such label would be more parsimonious than 'coverage procedure' when referring to such output.

Indeed, coverage $\left(2017^{12}\right)$ has the meaning of " 2 . the area that can be covered by a specified volume or weight of a substance", which brings to mind frequency distributions, both the theoretical sampling distributions underlying statistical models and the empirical sampling distributions that can be obtained by resampling. Thus, 'coverage intervals' apply to both.

Sampling distributions (e.g., sampling distributions of means) are based on the observed data provided by a sample, thereby centred on the sample's mean and dispersed as a function of the sample's variance. The typical $\mathrm{Cl}$ representation is rather descriptive of the underlying sampling distribution, with a central estimate and a dispersion amounting to a multiple of the standard error. Indeed, we could draw a normal curve by joining the resulting points as appropriate, thus representing the underlying sampling distribution, as Cumming (2012) proposes with his cat's-eyes figures. Such representation is a good summary of how Cls are produced: based on a sample, assuming the sample's statistics for the inference (thus, assuming $M=\mu_{x}=\mu$, thus $H_{R}$ ), generatingin theory or practice-the corresponding sampling distribution under such assumption, and partitioning the sampling distribution so that the resulting interval covers a given percentage of such distribution (Smithson, $2003^{13}$ ).

Of course, we are not interested in describing sampling distributions but on estimating parameters given a margin of error. Thus, it seems reasonable to assume that the population parameter ought to be not exactly where the sample statistic is but within the range of the interval, with X\% probability. It also seems reasonable to assume that all data values should have a given probability under the distribution, with values closer to the mean being more probable as true estimates than values further away (Cumming, 2012). Such assumptions, however, would be wrong.

Firstly, the sampling distribution is the procedure, based on one observed sample, and because there is just one distribution, all possible values, whether observed or not, ought to have a location under such distribution (i.e., the sampling distribution is one, thus exhaustive).

Secondly, given the exhaustive sampling distribution, coverage-based estimation is based not on the observed data but on the observable random data (Neyman, $1977^{14}$ ), and the confidence coefficient

\footnotetext{
${ }^{12}$ Coverage. (2017). In Oxford Dictionaries. Retrieved from https://en.oxforddictionaries.com/definition/coverage

${ }^{13}$ Smithson, M. (2003). Confidence intervals. Thousand Oaks, CA: Sage.

${ }^{14}$ Neyman, J. (1977). Frequentist probability and frequentist statistics. Synthese, 36(1), 97-131.
} 
is not the probability of the parameter falling within the interval but "of the [confidence bounds] falling on both sides of a specified [statistic]" (Neyman, 1941, p. $133^{15}$ ). More simply, we can consider coverage-based estimation as based on an infinite set of counterfactuals related to the samples that we did not observe. We can create such counterfactuals following a principle of symmetry or reciprocity, as follows: If a given value in the distribution is at a certain distance from the mean, then it stands to reason that the mean is also at the same distance from that value, as it would be that such distance would remain unchanged if the mean happened to be that value. Equally, if an $\mathrm{X} \%$ of the values in the distribution is contained within an interval, it stands to reason that the mean will also be contained within similarly sized intervals if the mean happened to be any of those alternative $\mathrm{X} \%$ values. Ergo, there is an $\mathrm{X} \%$ frequentist chance that the population value is within the interval. Thus, the inference is not based on the statistic being an approximated or best estimate for the parameter-indeed, "[the] probability must be calculated under the assumption that [the statistic] is the true value of [the parameter]" Neyman, $1937^{16}$, p. 348 -but on the counterfactual that the sample current distribution also appears under an infinite number of alternative distributions. Therefore, a coverage interval is not only a mere descriptive of the actual sample but also covers $\mathrm{X} \%$ of alternative values which, conditioned on the statistic, may be better estimates of the parameter than the statistic is.

The Fundamental Confidence Fallacy kicks in when we forget that we are talking about frequentist probability, thus $\mathrm{X} \%$ of possible estimates conditioned on this sample shall be found within the limits, but also $100-X \%$ of possible estimates shall be found outside the limits. The true parameter may as well be one of the latter without us being the wiser.

Thirdly, the confusion of sampling distributions (as procedures) with coverage intervals (as outputs) largely explains Morey et al.'s precision fallacy. The width of a coverage interval has to do with the width of the standard error of the sampling distribution, and a smaller error may as well be a sign of precision as long as we do not forget that we are still talking about a sample-bound estimate. Said otherwise, a small interval may as well be precisely wrong. Greater precision in the form of a smaller standard error in the sample is largely achieved via increasing sample size, which has by itself a dual effect: It provides greater opportunities for the sample mean to be more representative of the population mean via the strong law of large numbers (Spanos, 2013), and it also reduces the variance, thus the standard error, in the sample. Precision depends not only on smaller intervals but also on more representative samples.

A coverage interval, as output of a coverage procedure, simply indicates a shorter range of estimated values, not necessarily more accurate or precise estimation of the central location for a parameter; however, we may certainly not discard a correlation between precision at procedure level and coverage interval length (e.g., Neyman, 1977).

Fourthly, the counterfactual explanation also brings home another important issue: All values, inside and outside the distribution, have the same potential to be the true value of the parameter. In fact, a representation of such counterfactual would be a uniform distribution. Therefore, the sampling

\footnotetext{
${ }^{15}$ Neyman, J. (1941). Fiducial argument and the theory of confidence intervals. Biometrika, 32(2), 128-150.

${ }^{16}$ Neyman, J. (1937). Outline of a theory of statistical estimation based on the classical theory of probability. Philosophical Transactions of the Royal Society of London. Series A, Mathematical and Physical Sciences, 236(767), 333-380.
} 
distribution of a single sample cannot possibly represent the probable distribution of the parameter, as Cumming $\left(2012^{17}\right)$ suggests with his cat's-eyes. On a coverage interval, the true value of the parameter may be inside or outside the interval with equal plausibility, although, given argument two above, the expectation is that it will be one of those X\% inside the interval. As Bowley, possibly paraphrasing Fisher (e.g., $1959^{18}$ ), put it, "either an improbable event has occurred or the [parameter] in the population is within the limits" $\left(1934^{1920}\right.$, p. 609).

Fifthly, the sampling distribution can also play a double role as a test of significance. Data under the sampling distribution have their appropriate probabilities under $\mathrm{H}_{R}$, and 'zero' can be assessed against such distribution. Thus, whenever 'zero' is outside the interval, we can say that it has low probability under $\mathrm{H}_{\mathrm{R}}$. Alternatively, we could say that the test is significant at the $100-\mathrm{X} \%$ coverage level. We cannot forget, however, that the test is based on assuming the statistic as the parameter $\left(H_{R}: M=\mu x=\mu\right)$, which does not necessarily lead to recognise it to be true, nor any value to be more or less plausible, credible or reasonable other than under $\mathrm{H}_{\mathrm{R}}$. Again, the only conclusion we could possibly achieve is, either a rare event occurred or ' 0 ' is not the population parameter. More easily, as Neyman proposed, decide to behave as if ' 0 ' is not a true value for the parameter, without attaching any probability to its certainty or plausibility. Because of the dichotomous nature of a test and because the test is based on the boundaries of the sampling distribution as cut-off points, $\mathrm{Cls}$ can certainly substitute the sampling distribution for the purpose of significance testing (e.g., Mayo, $2012^{21}$ ).

All above points coalesce into a single summary point: Cls as currently represented are more descriptive of the underlying sampling distribution (mean and a multiple of the standard error) than of an inferential coverage interval. A coverage interval proper should be represented without a central estimate; i.e., as a bounded range of values on a uniform distribution, such range having $\mathrm{X} \%$ expectation (Neyman, 1977) of containing the true parameter.

\section{Countable intervals}

"On a given sample, we could get any one of the possible confidence intervals. If $95 \%$ of the possible confidence intervals contain the true value ... it seems reasonable to say that we have $95 \%$ certainty that we obtained one of the confidence intervals that contain the true value" (Morey et al., 2016, p. 104, 105).

A countable interval is the realization of the frequentist probabilities of a coverage procedure in the long-run (Neyman, 1941). This is the typical serial display of coverage intervals showed in

\footnotetext{
${ }^{17}$ Cumming, G. (2012). Understanding the new statistics. Effect sizes, confidence intervals, and meta-analysis. New York, NY: Routledge.

${ }^{18}$ Fisher, R.A. (1959). Statistical methods and scientific inference (2nd ed). Edinburgh, UK: Oliver and Boyd.

${ }^{19}$ Neyman, J. (1934). On the two different aspects of the representative method: the methods of stratified sampling and the method of purposive selection. Journal of the Royal Statistical Society, 97(4), 558-625.

${ }^{20}$ Bowley. (1934). Discussion on Dr. Neyman's paper. [Peer commentary on the paper "On the two different aspects of the representative method: the methods of stratified sampling and the method of purposive selection" by J. Neyman (1934)].

${ }^{21}$ Mayo, D. (2012). Statistical science meets philosophy of science part 2: shallow versus deep explorations. RMM, 3, 71-107.
} 
simulations, so that we may ascertain the long-run probability of the procedure (e.g., Neyman, 1977; Cumming, 2012). The main difference between a coverage procedure and a countable one is that a coverage procedure covers frequency distributions while a countable one counts 'successful' coverage intervals. In a nutshell, a countable procedure is binomial. In simulations, it is easy to count the frequency of coverage intervals that succeed in capturing the parameter (alternatively, the frequency of those that fail to do so). (Notice that an X\% countable interval may, perhaps, also allow for a meta-analytical approach by using "the common part as some kind of 'summary' [coverage] interval”-Neyman, 1977, p. 120; also Smithson, 2003).

We can see the conflict between a coverage interval and a countable interval in Morey et al.'s argument regarding the Fundamental Confidence Fallacy, defined in a manner which is acceptably consistent with a coverage interval (as a lower nested definition) but reasoned out as a countable interval. The reasoning is not wrong in itself; only not parsimonious. However, the long-run explanation helps where the coverage explanation may fail. That is, we cannot know whether a particular coverage interval captures the true parameter or not, but we can trust that, in the long run, it will fail to do so $100-X \%$ of the time (Neyman, 1977). Thus, an X\% countable procedure rests on the assumption that $\mathrm{X} \%$ of coverage intervals will capture the true parameter within their $\mathrm{X} \%$ coverage interval, thus resulting in an $\mathrm{X} \%$ countable interval.

The Fundamental Confidence Fallacy comes into play if we take for granted that a particular interval ought to be one of those $\mathrm{X} \%$, or if claiming that we are $\mathrm{X} \%$ certain it is one of the true intervals.

The conflict between coverage and countable intervals also appears in the section explaining Neyman's theory of confidence intervals and alluding to the long-run, power and the control of error rates. The close relationship with data testing is illustrative. However, Neyman never really defined his confidence intervals as procedures in the long-run. Instead, his definition accords to a statistical model-based coverage interval which can be appropriately corroborated in the long-run (e.g., 1937). As for controlling error rates, Neyman expects a Type I error rate in the long run by a consistent use of a given coverage coefficient (e.g., 1977), but there is no Type II error entering the procedure, thus error rate control is rather passive. Finally, lacking Type II error, there is no power in the procedure either. Namely, Morey et al., even Neyman, discuss confidence intervals using constructs from acceptance testing (power, error control, exclusion of false values, long-run, behavioural decisions, etc.) but such constructs are often metaphorical.

\section{Credible intervals}

"Bayesian reasoning [evolves] from prior and likelihood to posterior and so-called credible intervals ... The credible interval can be interpreted as having [X\%] probability of containing the true value, because the values within it account for [X\%] of the posterior [distribution]" (Morey et al., 2016, p. 113)

Credible intervals are succinctly, albeit clearly, defined by Morey et al.: based on prior information and observations from the actual data, a posterior distribution is generated, the credible interval being but a descriptive of the coverage $\mathrm{X} \%$ of this posterior distribution. Therefore, unlike sampling distributions, the posterior distribution is not the procedure but the actual output, and the resulting 
interval can, indeed, be represented as a distribution. A credible interval therefore inherits properties inherent to a distribution, such as that the interval provides $\mathrm{X} \%$ certainty of containing the true parameter, that a central estimate is the most probable value while a range of nearby values are also plausible with decreasing probability, and that a shorter interval equals greater precision in the estimation.

\section{Final notes}

Despite what it has been said above, we should also consider at which point the discussion veers into distinctions without a difference-not so much as formal logical fallacies but rather as "linguistic or conceptual distinctions of no practical importance" (Distinction without a difference, 2016 ${ }^{22}$; also Taleb, $2010^{23}$ ). Four distinctions seem debatable. Firstly, the distinction between procedure and output. Researchers estimating by interval are plausibly more interested in the interval than in the procedure, therefore assuming that an $\mathrm{X} \%$ interval subsumes whatever procedure produced it. Secondly, the distinction between output and outcome. Researchers estimating by interval plausibly use an interval as an inferential statistic for an unknown (and unknowable) parameter, an X\% interval being an expectation of where the parameter may be rather than a measure of final precision regarding whether it will ultimately be in the interval or not. Thirdly, the current depiction of a coverage interval. Researchers estimating by interval plausibly interpret an interval as the expected range of values for the unknown parameter, not as a (posterior) frequency distribution (Cumming, 2012, 2014 ${ }^{24}$, being a clear exception). Therefore, the depiction of a central estimate, albeit inaccurate, may simply double as a description of the sample. (Such depiction is correct for representing credible intervals, however.) Fourthly, the distinction between coverage intervals and credible intervals when using uniform priors. Both lead to the same numerical outputs and, thus, same intervals. Under such condition, a coverage interval has the same 'confidence' than a credible interval when the latter is also interpreted as a range of plausible values rather than as a probability distribution.

A second note of interest is to caution about the misleading environment that simulations provide. Simulations lead to the easy confusion between coverage intervals and countable intervals without appreciating the nesting of the former within the latter, thus to non-parsimonious definitions.

Simulations also lead to a fallacy of hindsight reasoning, namely because we know where the parameter lies (a.k.a., the outcome), so it is easy to ascertain which interval captures it or not, and with what precision. In real research, however, we do not have such prerogative; for example, I do not know what to gain from Morey et al.'s Figure 1, as I can see where the submarine is as well as I can appreciate that most intervals are equally bad at capturing the hatch in $A$ and equally good at capturing the hatch in B. (Add to it that there is only one chance to save the crew and no procedure to decide which portion of the interval to use-therefore it is reasonable to assume the decision would be to use the centre of the selected interval-in which case parameter estimation is more

\footnotetext{
${ }^{22}$ Distinction without a difference. (2016). In Wiktionary, The Free Dictionary. Retrieved from https://en.wiktionary.org/wiki/distinction_without_a_difference

${ }^{23}$ Taleb, N. N. (2010). The black swan (2nd ed.). New York, NY: Random House.

${ }^{24}$ Cumming, G. (2014). The new statistics: why and how. Psychological Science, 25(1), 7-29. doi: $10.1177 / 0956797613504966$
} 
relevant than estimation by interval, the crew would be dead under any of the intervals in Fig.1A and it would be saved under any of the intervals in B.)

In summary, Morey et al. (2016) have done good work in clarifying the differences between frequentist intervals and Bayesian intervals, in highlighting fallacies in the interpretation of frequentist intervals, and in simulating differences among several estimations by intervals. Even when I point out to other differences, recognise that some distinctions may have little bearing in practice and warn about the fallacy of hindsight attached to simulations, I believe Morey et al.'s theoretical review makes for good reading and should form part of methodology training. 